\title{
Calibration and Evaluation of an X-Ray Fluorescence Method for the Determination of Lead and Arsenic in Soils
}

\section{Dana J DiScenza ${ }^{1}$, Alison R Keimowitz ${ }^{2}$ and Neil Fitzgerald ${ }^{1 *}$}

${ }^{1}$ Department of Chemistry, Biochemistry and Physics, Marist College, Poughkeepsie, NY, USA

${ }^{2}$ Chemistry Department, Vassar College, Poughkeepsie, NY, USA

\begin{abstract}
A portable X-ray fluorescence method is described for the measurement of lead and arsenic in soils based on calibration with standard reference materials. Both elements exhibited good linear calibration curves. The method was used to analyze dried and ground soil samples obtained from the metropolitan New York City area. Results were compared to measurements obtained by graphite furnace atomic absorption spectrometry with microwave assisted acid leaching. No significant difference was found for lead measurements between the two methods however, arsenic values were significantly different with XRF exhibiting higher values. It is hypothesized that incomplete leaching and spectral interference are the predominant causes of this discrepancy. The XRF method provides a simple, quick, and nondestructive method for the determination of total lead and arsenic content in soils.
\end{abstract}

Keywords: X-ray fluorescence; Lead; Arsenic; Soil; Graphite furnace; Calibration

\section{Introduction}

X-Ray Fluorescence spectrometry (XRF) offers many advantages for environmental analysis of soils and sediments. XRF instruments require minimal sample preparation and are non-destructive, rapid, and portable. Numerous reports have been published demonstrating the application of XRF to the analysis of soils and sediments. A typical example was published by Chou et al. [1] who demonstrated the use of a portable XRF instrument for the assessment of lead and arsenic in the greater New Orleans area after Hurricane Katrina. Other relevant examples include the feasibility of using a portable XRF in the field for lead content in sieved soils [2], XRF analysis of arsenic contamination at an arsenic works site [3], and the application of portable XRF for heavy metal analysis of soils near abandon mines [4].

Disadvantages of XRF compared to routine laboratory instruments are the relatively high detection limits (generally in the part per million range) and inferior accuracy and precision. XRF also suffers from significant matrix effects which can make calibration problematic. Calibration methods for XRF include the use of matrix matched standards, adding an internal standard to the sample matrix, dilution to reduce matrix effects, and fusion of standards and samples with a suitable flux [5]. Calibration of modern commercial XRF instruments is often performed using calibration algorithms loaded into the instrument software. While this may be acceptable for in-situ monitoring work, it may not provide acceptable results compared to laboratory based methods. The United States Environmental Protection Agency (USEPA) state precision values between 1.24 to $29.2 \%$ relative standard deviation for ground and dried soils, depending on the element, with recoveries for Standard Reference Materials between 90 and 110\% [6]. Kristna et al. [7] compared XRF and Inductively Coupled Plasma Atomic Emission Spectrometry (ICP-AES) for the determination of heavy metals in soil samples. They used pressed powder pellets and empirical corrections to account for matrix effects in XRF and performed a total soil digestion with hydrofluoric acid prior to ICP-AES analysis. Using these methods good agreement was obtained. Kenna et al. [8] attempted to improve data quality of a field portable XRF by the use of soil and sediment Standard Reference Materials (SRMs) to calibrate the instrument. The reported measurements for Hudson River estuary sediment were in good agreement with independent laboratory analysis.

In this work we describe a variation of the calibration method described by Kenna et al. [8] using standard reference materials to calibrate a field portable XRF for the determination of lead and arsenic in soils obtained from a variety of locations in the New York City metropolitan area. The XRF method was compared to results obtained using Graphite Furnace Atomic Absorption (GFAA) after microwave assisted nitric acid leaching.

\section{Materials and Methods}

\section{X-Ray fluorescence}

X-Ray Fluorescence results were obtained on ground and dried soil samples obtained from the Rockaway Peninsula and Greenpoint, Queens, NY areas. The soils varied in composition from sandy to loamy. Lead and arsenic were measured using a field-portable XRF (Bruker Tracer III-V, Billerica, MA). Samples were dried at $30^{\circ} \mathrm{C}$ overnight, ground and placed into XRF sample cups. The XRF excitation source was set to $40 \mathrm{kV}$, at $25 \mu \mathrm{A}$ under 5 Torr vacuum. Data was collected for 2 minutes for each sample. The L1 line at $10.55 \mathrm{keV}$ was used for lead and Ka1 line at $10.54 \mathrm{keV}$ for arsenic. Calibration was performed by duplicate measurements of National Institute of Standards and Technology (NIST, Washington DC) Standard Reference Materials (Table 1). Chemically pure silica was used as a blank. The XRF signal intensity was plotted against the certified value for each SRM in order to construct calibration curves.

\section{Graphite Furnace Atomic Absorption (GFAA)}

Graphite Furnace Atomic Absorption (GFAA) spectrometry (Thermo M5, Walthan, MA) was used to compare measurements obtained using the XRF method. Instrument conditions are detailed in Table 2. Soil samples were prepared using a microwave digestion unit

*Corresponding author: Neil Fitzgerald, Department of Chemistry, Biochemistry and Physics, Marist College, Poughkeepsie, NY, USA, Tel: (845) 575-3000 ext. 2491; E-mail: neil.fitzgerald@marist.edu

Received January 09, 2014; Accepted March 29, 2014; Published March 31 2014

Citation: DiScenza DJ, Keimowitz AR, Fitzgerald N (2014) Calibration and Evaluation of an X-Ray Fluorescence Method for the Determination of Lead and Arsenic in Soils. J Environ Anal Chem 1: 103. doi:10.4172/jreac.1000103

Copyright: ( 2014 DiScenza DJ, et al. This is an open-access article distributed under the terms of the Creative Commons Attribution License, which permits unrestricted use, distribution, and reproduction in any medium, provided the original author and source are credited. 


\begin{tabular}{|l|l|l|}
\hline SRM & Kg) & (mg/Kg) \\
\hline 1646a (Estuarine Sediment) & 11.7 & 6.23 \\
\hline 1944 (NY/NJ Waterway Sediment) & 330 & 18.9 \\
\hline 2586 (Trace Elements in Soil) & 423 & 8.7 \\
\hline 2711a (Montana II Soil) & 1400 & 107 \\
\hline 2781 (Domestic Sludge) & 202.1 & 7.82 \\
\hline
\end{tabular}

Table 1: NIST standard reference materials used for XRF calibration.

\begin{tabular}{|l|l|l|}
\hline & Lead & Arsenic \\
\hline Wavelength $(\mathrm{nm})$ & 283.3 & 197.3 \\
\hline Slit Width $(\mathrm{nm})$ & 0.5 & 0.5 \\
\hline Matrix Modifier & Ammonium Nitrate & Nickel Nitrate \\
\hline Ashing Temperature $\left({ }^{\circ} \mathrm{C}\right)$ & 800 & 1200 \\
\hline Atomization Temperature $\left({ }^{\circ} \mathrm{C}\right)$ & 1200 & 2600 \\
\hline Calibration Method & External Standards & Standard Additions \\
\hline
\end{tabular}

Table 2: Graphite furnace atomic absorption operating conditions.

(CEM MARS, Charlotte, NC). Approximately $0.500 \mathrm{~g}$ of soil samples were combined with $10.00 \mathrm{~mL}$ of nitric acid (Fischer trace metal grade, Fairlawn, NJ) in microwave vessels. The microwave program involved heating to $175^{\circ} \mathrm{C}$ and holding at this temperature for 4 minutes 50 seconds. The vessels were left an appropriate time to cool before diluting with $18 \mathrm{M} \Omega$ water (Barnstead Nanopure, Lake Balboa, CA) to a volume of $100.00 \mathrm{~mL}$. The determination of arsenic required a standard additions method while external standard calibration was used for lead. Arsenic standards were prepared by appropriate dilutions of an atomic absorption standard solution (Ricca, Arlington, TX) with 10\% nitric acid. Lead standards were prepared by dilution of a lead standard solution (Fisher AA standard, Fairlawn, NJ) with 10\% nitric acid.

\section{Results and Discussion}

Calibration curves for lead and arsenic using standard reference materials as calibration standards are shown in Figures 1 and 2. In both cases SRM1646a (Estuarine Sediment) produced high signals that caused significant influences on the regressions and were omitted as outliers. The Pearson correlation coefficients ( $r^{2}$ values) including SRM1646a were 0.9180 and 0.5162 for lead and arsenic respectively. In the absences of 1646a, they improved to 0.9764 and 0.9946 for lead and arsenic respectively.

In order to validate the methods, a comparison to an accepted method was performed. In our laboratory Graphite Furnace Atomic Absorption spectrometry after microwave pretreatment is the standard method for elemental analysis and generally accepted to produce accurate results. A paired t-test indicated no significance difference for lead measurements between the two techniques at $95 \%$ confidence. For arsenic, however, the paired t-test indicates a significant difference between the two techniques at 95\% confidence with XRF values tending to be approximately double GFAA values. Figure 3 shows a comparison of XRF with GFAA for lead measurements. Ideally, if both techniques provide identical results, the data points would fit a linear regression line with a slope of one and a zero intercept. For lead results this is broadly true although XRF values tended to be higher on average than GFAA values. A similar trend is seen for arsenic with XRF measurements exhibiting significantly higher values than GFAA measurements in general (Figure 4). One soil value was removed as an outlier for lead measurements ( $\mathrm{r}^{2}$ was 0.4731 including outlier). Significant scatter is observed in both graphs which may be an indication of inherently poor precision caused by sample inhomogeneity as well as spectral interferences on the XRF measurements. In this work duplicate measurements of samples were seen to vary by between $1.9 \%$ and $69 \%$ for lead and between $26 \%$ and $170 \%$ for arsenic highlighting precision issues. Goldstein et al. [9] also observed a positive bias for XRF arsenic measurements in soils compared to ICP data after a microwave acid leaching procedure similar to the method described here. They note that "the XRF technique is measuring total amounts of analyte, whereas standard EPA methods are measuring only the nitric acid-digestible part of the soil and not the matrix". Akbulut et al. [10] noticed the same effect when comparing XRF measurement to ICPMS in soils. While they note that spectral interferences between arsenic and lead can affect accuracy and detection limits, they also state that incomplete leaching

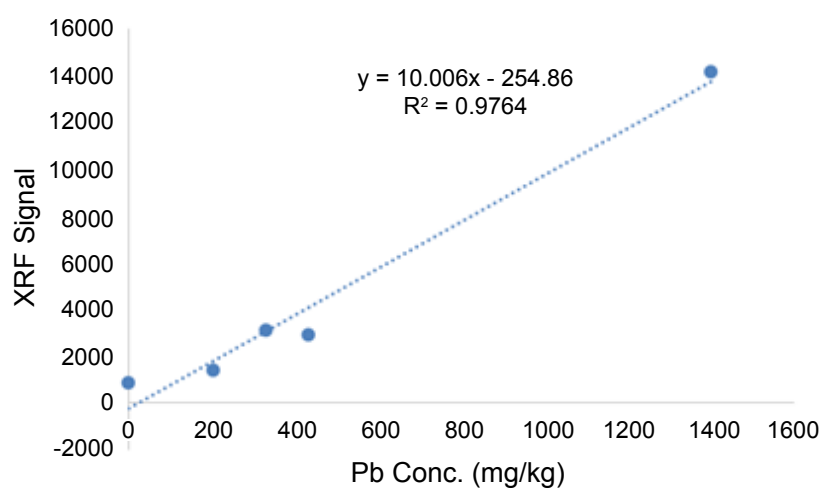

Figure 1: Calibration curve for lead by XRF.

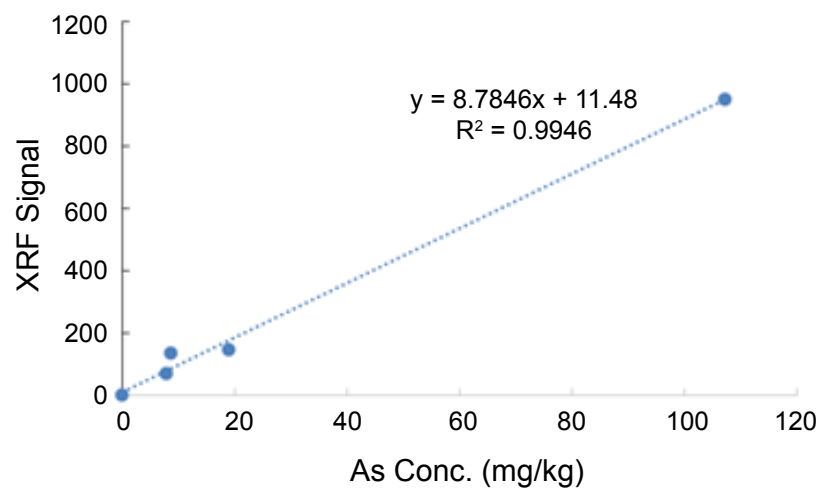

Figure 2: Calibration curve for arsenic by XRF.

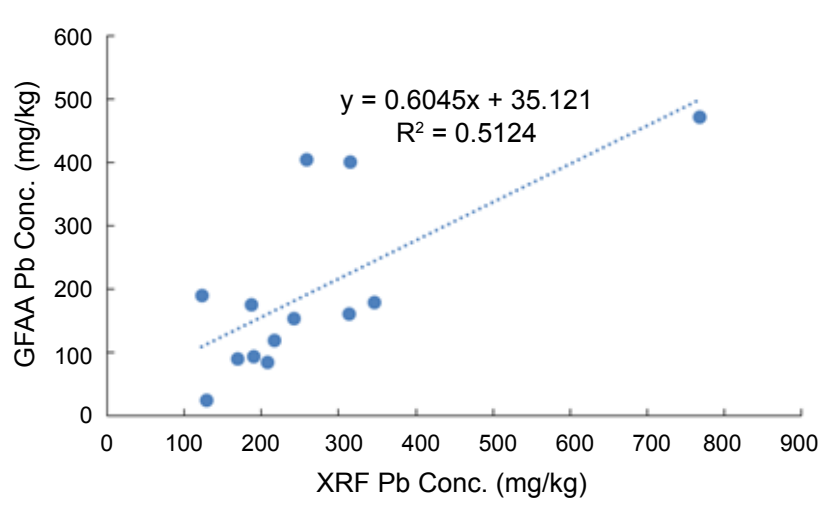

Figure 3: Comparison of XRF to GFAA for lead. 


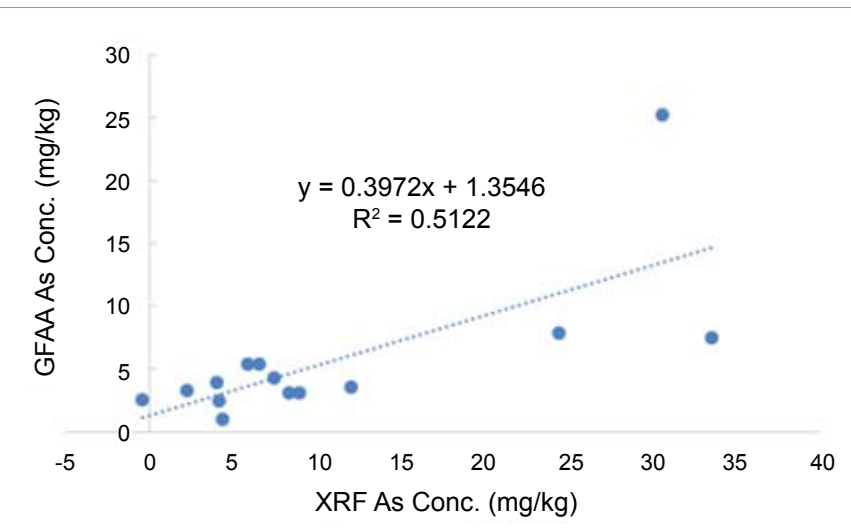

Figure 4: Comparison of XRF to GFAA for arsenic.

during the acid digestion process can lead to inaccurate results. Hassan et al. [11] studied microwave-assisted acid digestion methods and found that EPA method 3051, similar to the method used in this work, can result in recoveries as low as $47 \%$ for arsenic in reference materials. Recoveries were improved by modification of the method with the use of $\mathrm{HCl}$ but they note that disadvantages of using $\mathrm{HCl}$ may outweigh the advantages. It can be hypothesize that the higher arsenic levels in the standard reference materials used in this study to validate the GFAA method are more easily leachable by nitric acid than the arsenic strongly bound to the soil matrix in the samples. This is consistent with Goldstein's conclusion that "XRF and EPA methods are not directly comparable for relatively pristine soils" however "in contaminated soils where most of the analyte is nitric acid-leachable, EDXRF and standard EPA methods should give more similar results". Another explanation for the discrepancies is the well-known spectral interference of the lead line at $10.551 \mathrm{keV}$ on the arsenic line at $10.543 \mathrm{keV}$. While these lines are commonly used with mathematical deconvolution procedures to determine lead and arsenic, the instrument spectral resolution (145 $\mathrm{eV}$ ) suggests that significant overlap occurs. The calibration method used may account for interference effects to some extent by matrix matching, therefore it would be expected that spectral interference would have a greater influence on the precision than accuracy.

In conclusion, our data suggests that XRF using soil standard reference materials as calibration standards is an acceptable method for the determination of lead in dried and ground soils. XRF and acid leachable GFAA methods provide statistically similar results for lead but not for arsenic which we believe to be due to arsenic at relatively low concentrations tending to be strongly bound to the soil and not easily acid leachable. An alternative explanation is that it is an effect of uncompensated spectral interference although we believe this is more likely to affect precision than accuracy. Poor precision was observed for XRF measurements particularly for arsenic. Using soil standard reference materials offers a matrix matched calibration method and avoids needing to perform significant sample preparation or reliance on algorithms provided by the instrument manufacturer.

\section{Acknowledgement}

The authors wish to acknowledge Marist College, Vassar College, and individual contributions to a crowdfunding project on Rockethub for financial support. We wish to acknowledge Amy Mandigo (Marist College) for her help with the project.

\section{References}

1. Chou J, Elbers D, Clement G, Bursavich B, Tian T, et al. (2010) In situ monitoring (field screening) and assessment of lead and arsenic contaminants in the greater New Orleans area using a portable X-ray fluorescence analyser. J Environ. Monit 12: 1722-1729.

2. Markey AM, Clark CS, Succop PA, Roda S (2008) Determination of the feasibility of using a portable X-ray fluorescence (XRF) analyzer in the field for measurement of lead content of sieved soil. J Environ Health 70: 24-29.

3. Potts PJ, Ramsey MH, Carlisle J (2002) Portable X-ray fluorescence in the characterisation of arsenic contamination associated with industrial buildings at a heritage arsenic works site near Redruth, Cornwall, UK. J Environ Monit 4: 1017-1024.

4. Jang M (2010) Application of portable X-ray fluorescence ( $p X R F)$ for heavy metal analysis of soils in crop fields near abandoned mine sites. Environ Geochem Health 32: 207-216.

5. Skoog D, Holler F, Nieman T (1998) Principles of Instrumental Analysis (5thedn). Saunders College Publishing, Orlando, FI.

6. United States Environmental Protection Agency (1998) EPA Method 6200 Field Portable X-Ray Fluorescence for the Determination of Elemental Concentrations in Soil and Sediment. United States Environmental Protection Agency.

7. Krishna A, Mohan K, Murthy N, Govil P (2008) Comparative study of X-Ray Fluorescence and Inductively Coupled Plasma Optical Emission Spectrometry of heavy metals in the analysis of soil samples. Atomic Spectroscopy 29: 83-89.

8. Kenna T, Nitsche F, Herron M, Mailloux B, Peteet D, et al. (2011) Evaluation and calibration of a Field Portable X-Ray Fluorescence spectrometer for quantitative analysis of siliciclastic soils and sediments. J. Anal At Spectrom 26: 395-405.

9. Goldstein SJ, Slemmons AK, Canava HE (1996) Energy dispersive X-ray fluorescence methods for environmental characterization of soils. Environ Sci Technol 30: 2318-2321.

10. Akbulut S, Cevik U, Van AA, De Wael K, Van Grieken R (2014) Precision and accuracy of ST-EDXRF performance for As determination comparing with ICPMS and evaluation of As deviation in the soil media. Chemosphere 96: 16-22.

11. Hassan N, Rasmussen P, Dabek-Zlotorzynska E, Celo V, Chen H (2007) Analysis of environmental samples using microwave-assisted acid digestion and inductively coupled mass spectrometry: Maximizing total element recoveries. Water Air Soil Pollut 178: 323-334. 